\title{
Generation of infrared surface plasmon resonances with high refractive index sensitivity utilizing tilted fiber Bragg gratings
}

\author{
Tom Allsop, ${ }^{1}$ Ron Neal, ${ }^{2}$ Saeed Rehman, ${ }^{3}$ David J. Webb, ${ }^{1}$ Des Mapps, ${ }^{2, *}$ and Ian Bennion ${ }^{1}$ \\ ${ }^{1}$ Photonics Research Group, Aston University, Aston Triangle, Birmingham, B4 7ET, United Kingdom \\ ${ }^{2}$ School of Computing, Communications and Electronics, Faculty of Technology, University of Plymouth, Plymouth \\ PL4 8AA, United Kingdom \\ ${ }^{3}$ FiberLogix Ltd, Ashley House, Vale Industrial Park, Tolpits Lane, Watford, Herts WD18 9QP, United Kingdom
}

${ }^{*}$ Corresponding author: t.d.p.allsop@aston.ac.uk

Received 14 February 2007; revised 30 May 2007; accepted 31 May 2007;

posted 4 June 2007 (Doc. ID 80156); published 23 July 2007

\begin{abstract}
We demonstrate the use of tilted fiber gratings to assist the generation of localized infrared surface plasmons with short propagation lengths and a sensitivity of $d \lambda / \mathrm{dn}=3365 \mathrm{~nm}$ in the aqueous index regime. It was also found that the resonances could be spectrally tuned over $1000 \mathrm{~nm}$ at the same spatial region with high coupling efficiency (in excess of $25 \mathrm{~dB}$ ) by altering the polarization of the light illuminating the device. (C) 2007 Optical Society of America

OCIS codes: $060.2370,060.2310$.
\end{abstract}

\section{Introduction}

Gratings in fibers-including long period gratings (LPGs), fiber Bragg gratings (FBGs) and tilted fiber Bragg gratings (TFBGs) — and surface plasmon resonance (in both planar and fiber configurations) may all be used to detect changes in the refractive index of various substances with potential applications in chemistry, biochemistry and biology [1-4]. Surface plasmon resonance (SPR) is a prominent optical phenomenon that involves a resonant transfer of the pumping light energy to a surface-plasmon (SP) mode in the form of collective oscillations of electrons in the metal [5]. It is well known that SP generation is very sensitive to the polarization of the illuminating light, its wavelength and its angle of incidence on the metal surface which is used to detect index changes in biochemical/chemical reactions [6]. SPR biosensors offer the opportunity for real-time and label-free monitoring of biomolecular interactions [7]. The majority of SPR based systems operate in the visible or near infrared part of the

0003-6935/07/220001-05\$15.00/0

(C) 2007 Optical Society of America spectrum which gives a probe depth (the spatial extension of the SP into the surrounding environment from the surface of the metal) of around $200 \mathrm{~nm}$ to $300 \mathrm{~nm}$ [6]. The SPs exist at a metaldielectric interface and obey the following dispersion relation for two homogeneous semi-infinite media:

$$
\beta=k \sqrt{\left(\frac{\varepsilon_{m} \cdot n_{s}^{2}}{\varepsilon_{m}+n_{s}^{2}}\right)}=k n_{2} \sin (\xi),
$$

where $k$ is the free space wave number, $\varepsilon_{m}$ is the permittivity of the metal $\left(\varepsilon_{m}=\varepsilon_{m r}+i \varepsilon_{m i}\right), n_{s}$ is the refractive index of the sample to be tested, $n_{2}$ is the refractive index of the cladding and $\xi$ is the incident angle of the light onto the metal/dielectric interface which determines the wave-number projection onto that interface.

Generally fiber gratings comprise an axially periodic refractive index variation inscribed in the core of a photosensitive single-mode optical fiber by ultraviolet radiation, or other means, which couples light from the core to other core modes (FBG), to cladding modes (LPGs) or to some combination of core, cladding and radiation modes (TFBGs) at discrete wave- 
lengths [8]. There is a considerable body of published research relating to fiber grating based devices; with the majority of the these devices, the highest index sensitivity is obtained with test sample indices over $\sim 1.4$ [9] and they generally have less sensitivity in the aqueous index regime. Whilst the majority of work done with SPRs in a fiber configuration has been done in the visible-NIR wavelength range of $600 \mathrm{~nm}$ to $800 \mathrm{~nm}$, going to longer wavelengths may improve sensor performance [5] and reduce costs by making use of standard telecommunications components.

We report on a novel SPR fiber device utilizing a TFBG to enhance the coupling of the illuminating light to a SP generated on a silver coating applied to a lapped single mode fiber. It was also observed that the spectral location of maximum coupling to the SP was dependent upon the polarization state of the illuminating light and could be tuned from $1100 \mathrm{~nm}$ to $1700 \mathrm{~nm}$. Calculations performed on measurements from the experimental data suggest that the propagation length of these infrared SPs is short $(\sim 100 \mathrm{~nm})$ compared to the spatial extension (probe depth) from the silver/medium interface, which is around $1.5 \mu \mathrm{m}$. It was found that for a device that is still to be fully optimized, the maximum spectral index sensitivity $(\mathrm{d} \lambda / \mathrm{dn})$ was $3365 \mathrm{~nm}$ for the index range 1.335 to 1.370 (suitable for refractive index monitoring of aqueous solutions) for SPs generated from $1200 \mathrm{~nm}$ to $1700 \mathrm{~nm}$. Our results suggest that with an optimized fiber device, at least another order of magnitude increase in the sensitivity may be possible.

\section{Fabrication and Characterization}

The SPR fiber devices are constructed in three stages [10]. Firstly, the TFBG is written in a UV photosensitive single mode fiber using a uniform phase mask mounted on a goniometer and then tilted to a specific angle; labels are added to indicate the orientation of the tilted grating. Secondly, the fiber is lapped down to $10 \mu \mathrm{m}$ from the core-cladding interface. The labels on the fiber are used to indicate which region of cladding is to be removed so that the grating vector, the fiber axis and the normal to the lapped surface all lie in a plane (see Fig. 1). Thirdly, the flat of the lapped fiber is then coated with silver (thickness $35 \mathrm{~nm}$ ) using a sputter machine and mask.

The fiber devices are characterized with a broadband light source, the light being passed through a polarizer, a polarization controller and a polarization maintaining coupler before illumination of the sample, with the transmission spectra being monitored using an optical spectrum analyser (OSA) with a resolution of $0.005 \mathrm{~nm}$, and the polarization of the illuminating light via a second output arm of the coupler, see Figure 1. Initially a series of these devices was fabricated with angles of tilt from $1^{\circ}$ to $9^{\circ}$ degrees. It was found that it was possible to generate SPRs with all of them. For a single fiber device it was possible to produce SPRs over a large spectral range from $1200 \mathrm{~nm}$ to $1700 \mathrm{~nm}$, whilst the device was submerged in test sample fluids, see Fig. 2 (note that the features seen at maximum coupling of the SPR are artefacts due to the normalization procedure of the OSA and that the maximum coupling bandwidth can be considerably narrower. The polarization dependency of these SPR devices was investigated using the schematic shown in Fig. 1. The devices were submerged into various index matching solutions and the polarization of the illuminating light was changed. It was found that the maximum extinction induced by the SPR coupling is very much dependent upon the polarization of the illuminating light. It is expected that the coupling efficiency and spectral location of the SPR should dramatically change with polarization, with some polarization states resulting in low coupling efficiencies of only a few $\mathrm{dB}$ [11]. However, whilst variations of the coupling strength of the SPRs were observed with changes in polarization, our devices still produced large extinction ratios over the wavelength range studied, see Fig. 2. In Fig. 2 it can be seen that over the observed spectral range $(1220 \mathrm{~nm}$ to $1700 \mathrm{~nm})$, the device with a $7^{\circ}$ degree tilt angle exhibited extinction ratios in excess of $35 \mathrm{~dB}$ in a solution with a refractive index of 1.360 . It can also be seen that the maximum extinction ratio occurs with a surrounding medium with a refractive index of

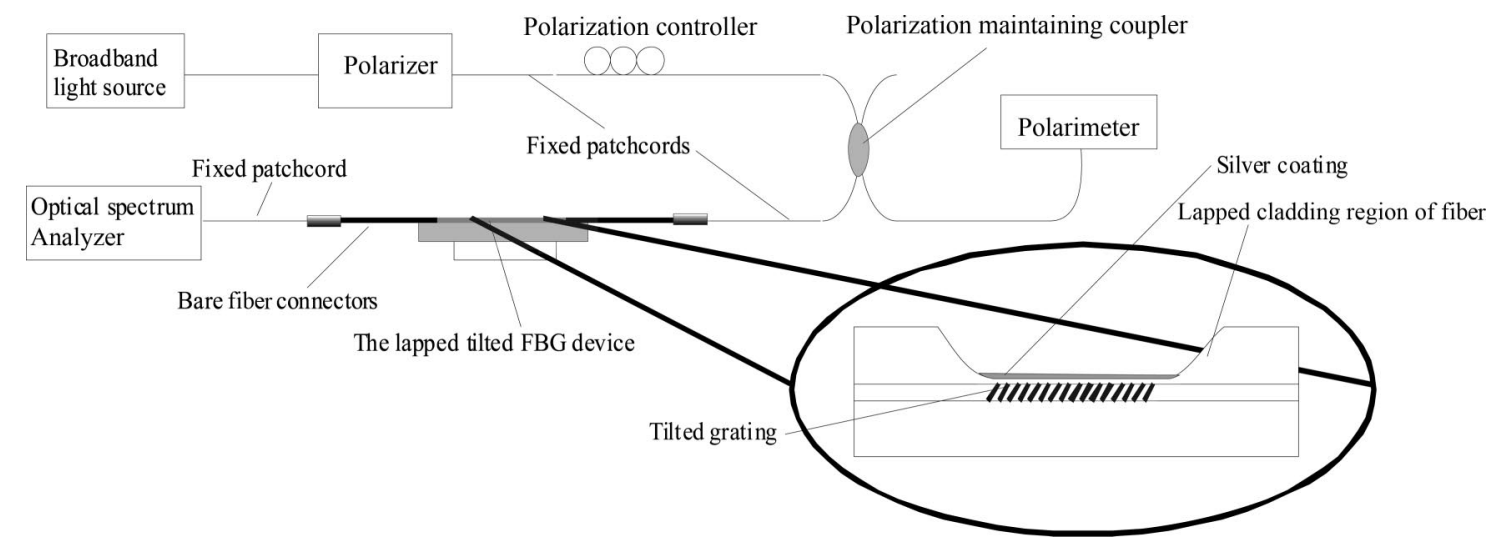

Fig. 1. Scheme used for the characterization of the tilted, lapped and coated fiber Bragg grating device. 

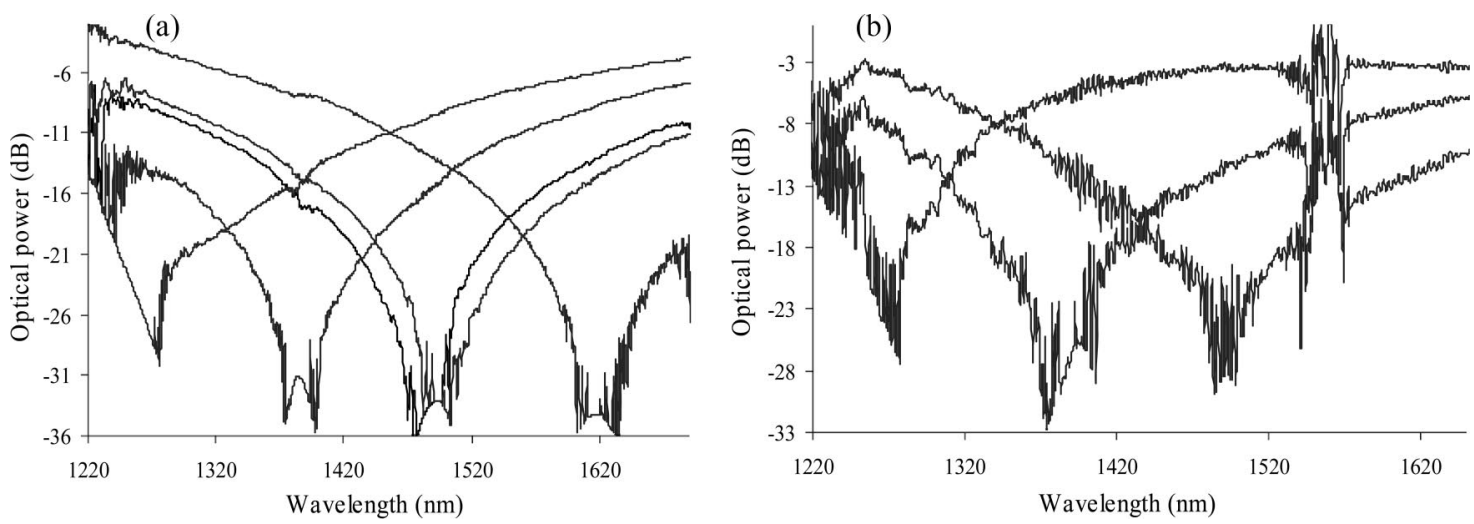

Fig. 2. The transmission spectra of fiber SPR devices illuminated with light of various polarization states. (a) Device in a solution with an index of 1.360 (Ag thickness $35 \mathrm{~nm}$, angle $7^{\circ}$, length $2.8 \mathrm{~cm}$ ). (b) Device in a solution with an index of 1.380 (Ag thickness $35 \mathrm{~nm}$, angle $3^{\circ}$, length $5.0 \mathrm{~cm}$ ).

around 1.36 with some reduction for both lower and higher refractive indices. It can also be seen from Fig. 2 that the extinction range of these devices ranges from around $1 \mathrm{~dB}$ to $35 \mathrm{~dB}$ for a given wavelength, as a function of polarization. It was also found that changing the tilt angle of the fiber Bragg grating changed the maximum extinction ratio achievable when altering the polarization of the illuminating light, see Fig. 3.

Comparing the coupling strength of the SPR with and without a grating (Fig. 3), it can be seen that the grating greatly enhances the SPR coupling in the aqueous index regime from $\sim 4 \mathrm{~dB}$ without grating to $25 \mathrm{~dB}$ for the 7 degree tilted grating with coupling increasing with increasing surrounding index to in excess of $35 \mathrm{~dB}$. It was also observed that spectral features in the fiber device with no grating were very much broader than with the gratings present. These spectral sensitivities obtained in the aqueous index regime are comparable to the highest sensitivities obtained with SPR based devices [3]; see Fig. 3(a). The highest spectral sensitivity obtained was $d \lambda / d n$ $=3365 \mathrm{~nm}$ from the 9 degree tilt angle device leading to a resolution (under the assumption of a $0.1 \mathrm{~nm}$ measurement resolution for the resonance wave- length) of $\sim 2 \times 10^{-5}$ over the index range of 1.34 to 1.38. For the fiber devices investigated to date, the spectral sensitivities varied from $700 \mathrm{~nm}$ to $1400 \mathrm{~nm}$ over the index region of 1.3 to 1.34 and from $2100 \mathrm{~nm}$ to $3400 \mathrm{~nm}$ over 1.34 to 1.38 . The power variation yielded results of $106 \mathrm{~dB}$ to $300 \mathrm{~dB}$ over the index region of 1.3 to 1.34 and $250 \mathrm{~dB}$ to $730 \mathrm{~dB}$ over 1.34 to 1.38 . The 7 degree tilted grating sensor achieves the strongest coupling to the SPR, with strengths varying from $10 \mathrm{~dB}$ to $+30 \mathrm{~dB}$ in the aqueous index regime. Using an atomic force microscope (AFM) it was found that the silver coating had an average thickness of $35 \mathrm{~nm}$ with a standard deviation of $\sim 6 \mathrm{~nm}$. Using this information along with the dispersion relationship for silver [12] and the cladding dispersion relationship of the D-shaped fiber, it was possible to reproduce similar transmission spectra to those of the SPR device for $p$-polarized light, by implementing Fresnel's equations for a three layered system for different refractive indices of the surrounding medium. The reflectivity $R$ of the fiber coating at various wavelengths for $p$-polarized light, with $E_{0}{ }^{p}$ the incoming and $E_{r}{ }^{p}$ the reflected field is given by expression 2 (see Ref. 6).
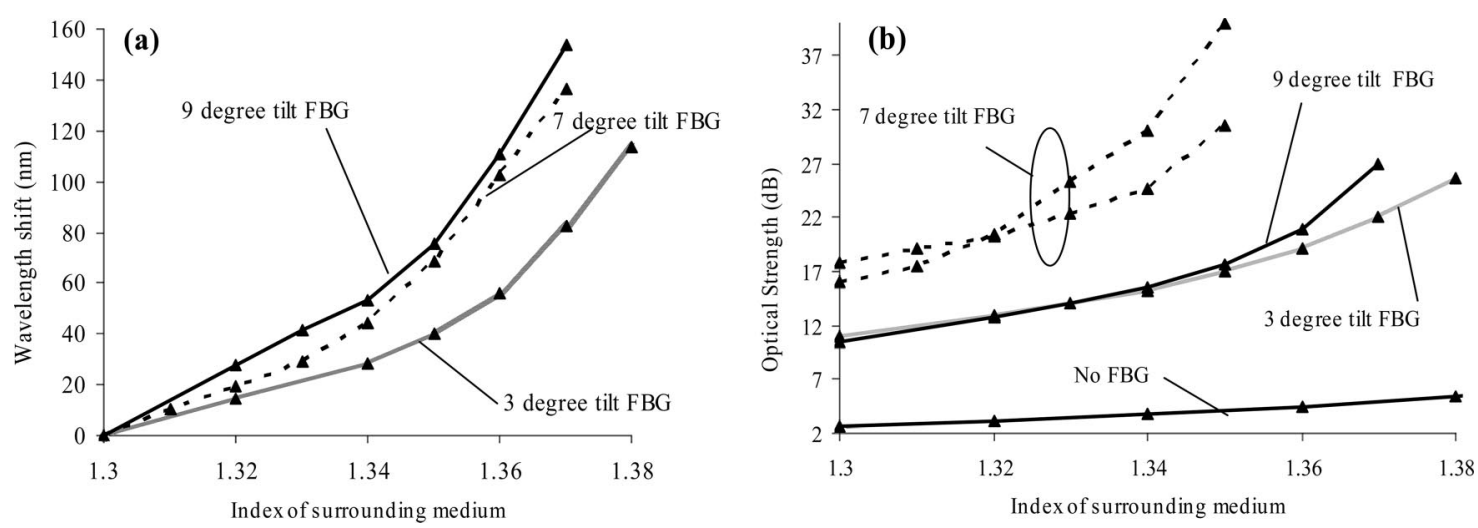

Fig. 3. The spectral characteristics of three devices with 3, 7 and 9 degree tilt angles. The two curves for 7 degrees correspond to two different incident polarizations. (a) Wavelength dependence and (b) strength dependence of the device as a function of the surrounding medium's refractive index. Also shown as a control in (b) is the coupling of a lapped and coated fiber with no grating inscribed. 

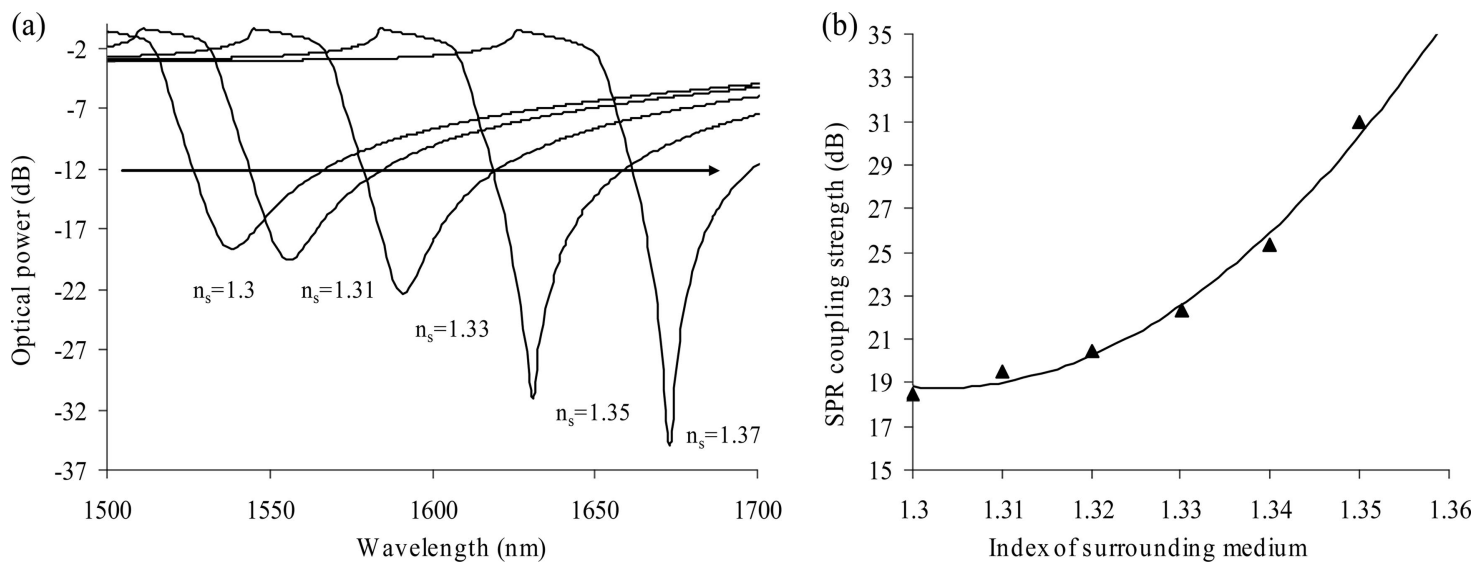

Fig. 4. (a) Theoretical transmission spectra of a SPR fiber device including a random variation of the thickness of the silver coating. (b) The theoretical coupling strength of the SPR as a function of surrounding index. Mean Coating thickness $35 \mathrm{~nm}$ with standard deviation of $6 \mathrm{~nm}$.

$R=\left|\frac{E_{r}^{p}}{E_{0}^{p}}\right|^{2}=\left|\frac{r_{n_{2} n_{m}}{ }^{p}+r_{n_{m} n_{s}}{ }^{p} \cdot \exp \left(2 i \mathbf{K}_{z, n_{m}} d\right)}{1+r_{n_{2} n_{m}}^{p} \cdot r_{n_{m} n_{s}}{ }^{p} \cdot \exp \left(2 i \mathbf{K}_{z, n_{m}} d\right)}\right|^{2}$

where $d$ is the thickness of the metal coating, and $r_{i, j}^{P}=\left(\frac{\mathbf{K}_{z, i}}{\varepsilon_{i}}-\frac{\mathbf{K}_{z, j}}{\varepsilon_{j}}\right) /\left(\frac{\mathbf{K}_{z, i}}{\varepsilon_{i}}+\frac{\mathbf{K}_{z, j}}{\varepsilon_{j}}\right)$ is the $p$-polarization amplitude reflection coefficients between layers $i$ and $j$ where the $\mathbf{K}$ s are the wave vectors of the illuminating incident light normal to each layer, and $\varepsilon_{j}$ are the permittivities of the $j$ th layer, $n_{m}$ is the metal index, $n_{s}$ is the index of the surrounding medium and $n_{2}$ is the index of the fiber cladding; for detail see [6]. Inspecting the results of the simulation plotted in Fig. 4 shows the bandwidths for indices of 1.35 and above to be considerably narrower than the experimental results shown in Fig. 2(a); $100 \mathrm{~nm}$ compared to $\sim 400 \mathrm{~nm}$. The width of the observed resonances suggests that these SPs have short propagation lengths, which may be exploited for some applications (SPR imaging techniques). To this end the propagation constants of the SP along the metal/dielectric interface were also calculated from the experimental data, thus giving some indication of the spatial localization of the SPs. The intrinsic loss $\left(\Gamma_{i}\right)$ of the fiber SPR device is based upon the optical properties of the materials used, and can be approximated using [13]

$$
\Gamma_{i}=\frac{n_{s}^{3} k_{0} \varepsilon_{i}}{2 \varepsilon_{r}^{2}}
$$

where $n_{s}$ is the refractive index of the test sample, $k_{0}$ is the free space propagation constant, $\varepsilon_{i}$ and $\varepsilon_{r}$ are the imaginary and real permittivities of the metal film. The radiative loss term $\left(\Gamma_{r}\right.$ which can be interpreted as an additional loss generated from light being reradiated into the cladding caused by surface roughness) can used to obtain the propagation constant of the SP. This loss term was estimated from experimental results such as those shown in Figure 1. Using the expression given by [13],

$$
W_{k}=2\left(\Gamma_{i}+\Gamma_{r}\right) /\left(n_{2} k_{0} \cos (\xi)\right),
$$

where $W_{k}$ is the width of the SPR at half maximum and $n_{2}$ is the index of the cladding and where $\xi$ is the angle of incidence on the metal coating of radiation emitted from the TFBG. The angle $\xi$ is calculated using the scattering angles associated with the various TM propagation constants $\left(n_{\beta}\right)$ generated by a D-shaped fiber with a silver coating, by the relationship given by the ray approach; $\sin (\xi)=n_{\beta} / n_{2}$. $\xi$ can be used to determine the projection of the incident wave-number along the metal/dielectric interface. Surface plasmons are generated when this wavenumber projection matches the dispersion relation of the plasmons given by expression 1. The TFBG enhances coupling to higher order $\mathrm{TM}_{v}$ modes to produce a larger range of scattering angles than in multimode or circular cladding single mode fiber [14]. These propagation constants and their associated coupling coefficients are calculated using a dispersion relationship derived in [15]. It was found that only a small number of modes contributed significantly to the SP generation (work to be published).

The radiative loss term $\left(\Gamma_{r}\right)$ is a quantity that is used to obtain the propagation length; $L_{x}$ (which yields an estimate of spatial resolution) of the SP via the characteristic propagation constant [6] which is defined for a non-smooth surface as:

$$
L_{x}=1 / 2\left(\operatorname{Im}\left(k_{0} \sqrt{\frac{\varepsilon_{m} \cdot n_{s}^{2}}{\varepsilon_{m}+n_{s}^{2}}}\right)+\Gamma_{r}\right) .
$$

The experimentally observed width of the resonance is much larger than that expected from the intrinsic loss alone; in the absence of other factors this suggests that the propagation lengths along the metal/ dielectric interface are short, ranging from $40 \mathrm{~nm}$ to $120 \mathrm{~nm}$, see Fig. 5. This may be contrasted with typical values from smooth surfaces [3], which range from $50 \mu \mathrm{m}$ to $150 \mu \mathrm{m}$ and have a spectral width at half maximum of just a few nanometres. Further 


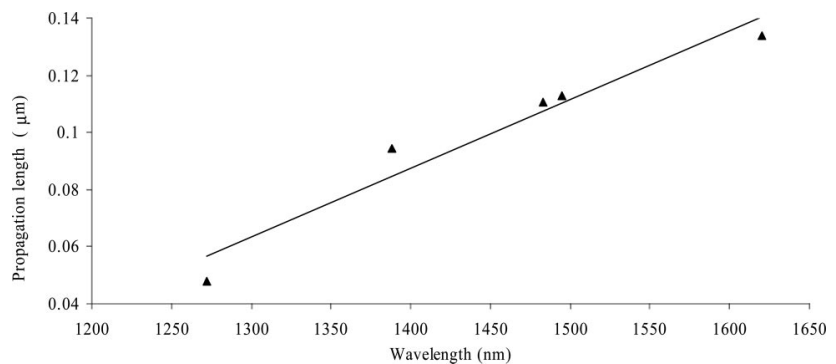

Fig. 5. The characteristic propagation length of the surface plasmons as a function of wavelength.

work is clearly required to fully explain this behaviour. These propagation lengths are of similar dimensions to the granularity of the silver surface observed by AFM, which suggests that these devices are producing highly spatially localized surface plasmons. Furthermore, calculations to reproduce the transmission spectra of the SPR devices suggest that for different polarization states, SPs are being generated at the same spatial location of the SPR fiber device but with different resonant wavelengths (work to be published). This suggests that the spatial extension of the SP evanescent fields (E-field component normal to the metal/dielectric interface) can be controlled at the same spatial location on the SPR fiber device by controlling the polarization of the illuminating light.

\section{Conclusion}

We have demonstrated a novel SPR fiber device utilizing a tilted fiber Bragg grating to enhance the coupling of the illuminating IR light to spatially localized surface plasmon resonances on a silver coated lapped single mode fiber. It was found that by altering the polarization of the light the resonance could be tuned over the spectral range from $1100 \mathrm{~nm}$ to $1700 \mathrm{~nm}$ with extinction ratios in excess of $35 \mathrm{~dB}$ for the aqueous index regime (1.34 to 1.37). Also the polarization dependence can control the spatial extension of the SPR from the metal/dielectric interface at a given location.

\section{References}

1. S. A. Vasiliev and O. I. Medvedkov, "Long-period refractive index fiber gratings: properties, applications, and fabrication techniques," Proc. SPIE 4083, 212-223 (2000).

2. K. Schroeder, W. Ecke, R. Mueller, R. Willsch, and A. Andreev, "A fiber Bragg grating refractometer," Meas. Sci. Technol. 12, 757-764 (2001).

3. M. Piliarik, J. Homola, Z. Mankova, and J. Ctyroky, "Surface plasmon resonance sensor based on a single-mode polarization-maintaining optical fiber," Sens. Actuators B 90, 236-242 (2003).

4. J. Homola, "Surface plasmon resonance sensors: review," Sens. Actuators B 54, 3-15 (1999).

5. S. Patskovsky, A. Kabsahin, M. Meunier, and J. Luong, "Properties and sensing characteristics of surface plasmon resonance in infrared light," J. Opt. Soc. Am. A 20, 1644-1650 (2003).

6. H. Raether, Surface Plasmons on Smooth and Rough Surfaces and on Gratings (Academic, New York, 1997).

7. J. M. Brockman, B. Nelson, and R. Corn, "Surface plasmon resonance imaging measurement of ultra-thin organic films," Annu. Rev. Phys. Chem. 51, 41-63 (2000).

8. T. Erdogan, "Fiber grating spectra," J. Lightwave Technol. 15, 1277-1292 (1997).

9. M. Iga, A. Seki, and K. Watanabe, "Gold thickness dependence of SPR-based hetero-core structured optical fiber sensor," Sens. Actuators B 106, 363-368 (2005).

10. T. Allsop, R. Neal, S. Rehman, C. Zhang, D. Webb, D. Mapps, and I. Bennion, "Surface plasmon resonance generation utilizing gratings for biochemical sensing," in Proceedings of the Eighteenth International Conference on Optical Fiber Sensors (Optical Society of America, Washington, D.C., 2006), paper WA4.

11. J. Homola and S. Yee, "Novel polarization control scheme for spectral surface plasmon resonance sensors," Sens. Actuators B 51, 331-359 (1998).

12. E. D. Palik and G. Ghosh, Handbook of Optical Constants (Academic, San Diego, 1998).

13. E. M. Yeatman, "Resolution and sensitivity in surface plasmon microscopy and sensing," Biosens. Bioelectron. 11, 635-649 (1996).

14. R. C. Jorgenson and S. Yee, "A fiber-optic chemical sensor based on surface plasmon resonance," Sens. Actuators B 12, 213-220 (1993).

15. C. Tsao, Optical Fiber Waveguide Analysis (Oxford, New York, 1992). 